\title{
Outcomes and Risk Factors Associated with Drug Resistant Tuberculosis in Rural Eastern Cape, South Africa
}

\author{
Carine Bokop Fotso, Sandeep D. Vasaikar and Teke Apalata \\ Division of Medical Microbiology, Department of Laboratory-Medicine and Pathology, Faculty of Health Sciences, \\ Walter Sisulu University and National Health Laboratory Services, Mthatha, SA
}

\author{
Article history \\ Received: 5-3-2018 \\ Revised: $13-06-2018$ \\ Accepted: 10-08-2018 \\ Corresponding Author: \\ Teke Apalata \\ Division of Medical \\ Microbiology, Department of \\ Laboratory Medicine and \\ Pathology, Faculty of Health \\ Sciences, Walter Sisulu \\ University, Mthatha, Eastern \\ Cape, South Africa \\ Email: ruffinapalata@gmail.com \\ tapalata@wsu.ac.za
}

\begin{abstract}
With the emergence of Multidrug-Resistant (MDR) and Extensively Drug-Resistant (XDR) strains of Mycobacterium tuberculosis, Tuberculosis (TB) has become an even greater threat in South Africa (SA). The study sought to investigate the resistance pattern of anti-TB drugs in M. tuberculosis as well as to determine the associated risk factors and outcomes for MDR-TB and XDR-TB. A descriptive study was conducted in 2 sub-districts of Eastern Cape province. Laboratory testing on sputum samples was done using genotypic and phenotypic methods. Data related to risk factors and outcomes were collected. SPSS v.22 was used for statistical analyses. 224 patients were enrolled (33.9\% HIV negative and $66.1 \%$ HIV positive). Of 224 TB isolates obtained, 196 (87.5\%) were MDR-TB (including 23 rifampicin mono-resistant isolates), 2 $(0.9 \%)$ were isoniazid mono-resistant and 26 (11.6\%) were Pre-XDR and XDR-TB. Of 196 MDR-TB patients, 143 (72.9\%) had a favourable outcome post anti-TB therapy as compared to $17(65.4 \%)$ XDR-TB patients out of 26; but this difference was not statistically significant $(\mathrm{OR}=1.5$; CI: $0.6-3.4 ; \rho=0.27)$. Whilst $118(68.6 \%)$ out of 172 MDR-TB cases were newly diagnosed, previous exposure to anti-TB therapy was found to be a significant risk factor for developing XDR-TB $(\mathrm{OR}=2.5 ; \mathrm{CI}=1.1-5.9 ; \rho$ $=0.023)$. Despite that $34(26.2 \%)$ out of 130 new drug-resistant (DR)-TB patients completed their therapy, only $17(13.1 \%)$ were declared cured as compared to $23(33.8 \%)$ cured patients among the previously treated group $(\rho<0.0001)$.In contrary, $9(13.2 \%)$ cases of relapse and $11(16.2 \%)$ defaulters were found among the previously treated DR-TB patients as compared to new cases $[1(0.8 \%)$ relapse versus $11(8.5 \%)$ defaulters, respectively] on therapy $(\rho<0.0001)$. High rates of DR-TB among treatmentnaive patients, compounded with prevalent cases of relapses and defaulters among previously treated patients, raise the importance of DR-TB acquisition through direct transmission rather than the evolution of resistance.
\end{abstract}

Keywords: Multidrug-Resistant, Extensively Drug-Resistant, Outcomes, Risk Factors, Favourable Outcomes, Poor Outcomes

\section{Introduction}

First discovered by Robert Koch (1882), Mycobacterium tuberculosis (M.tb) is a pathogenic bacterial species in the family Mycobacteriaceae and the causative agent of most cases of tuberculosis (TB) (Ryan and Ray, 2004). The bacteria usually infect lungs, but TB bacteria can infect any part of the body such as the kidney, spine and brain. If not treated properly, TB disease can be fatal (CDC. CDC 24/7: Saving Lives Protecting People, 2016). According to World Health
Organization (WHO), tuberculosis is the most important resurgent disease worldwide, with the highest morbidity and mortality rates of any single pathogen (WHO, 2006). Worldwide, there are two million deaths per annum from TB and it is estimated that a third of the world's population are latently infected with M.tb (2).

There are currently 22 countries that are referred to as the TB "high burden" countries (HBCs) and they had been prioritized at a global level since 2000. These countries accounted for $82 \%$ of all estimated cases of TB worldwide in 2013 (WHO, 2015). Among the HBCs, 
South Africa (SA) has the third highest absolute number of reported incident cases and the fifth highest number of estimated prevalent cases of active TB (WHO, 2012). After adjusting for population size, SA has the highest incidence and prevalence of TB among the HBCs (860/100 000) (Bateman, 2015). It also has the largest number of HIV-associated TB cases and the secondlargest number of diagnosed multidrug-resistant (MDR)TB cases (after India) (WHO, 2012). According to Statistics South Africa (STATSSA, 2013), TB was the leading cause of death in South Africa with over 40,542 $(8.8 \%)$ deaths notified. With the emergence of multidrug-resistant (MDR) and extensively drugresistant (XDR) strains of M.tb, TB has become an even greater threat (WHO, 2012). With almost 500000 MDRTB cases emerging each year worldwide, between $5 \%$ and $7 \%$ of them are becoming XDR-TB (WHO, 2010).

The emergence of MDR-TB and XDR-TB globally has raised alertness and concern about incurable forms of TB. While the 2012 WHO Global TB report documents progress in TB control, it also describes the response to MDR-TB as inadequate and slow. Only 30 of 107 countries reporting MDR-TB treatment outcomes achieved the target treatment success rate of $\geq 75 \%$ (WHO, 2012).

Drug resistant TB (DR-TB) had emerged in South Africa by the 1980s, but was not thought to be a major problem (Schaaf et al., 1996). In 2014, WHO estimated that $3.3 \%$ of new cases and $20 \%$ of previously treated cases of TB were MDR-TB (WHO, 2015). Dheda et al. (2010) reported that the majority of new drug resistant TB (DR-TB) cases diagnosed in SA were due to transmission of already resistant strains. At present it is estimated that only $2 \%$ of MDR-TB cases worldwide are being diagnosed and treated appropriately, mainly because of inadequate laboratory services. Drug-resistant tuberculosis (DR-TB), particularly multi-drug resistant tuberculosis (MDR-TB) and extensively-drug resistant tuberculosis (XDR-TB), represent significant threats to global TB control efforts and subsequently a major public health concern in several countries (WHO, 2017). The World Health Organization recommends the use of rapid molecular tests Xpert ${ }^{\circledR}$ MTB/RIF assay (Cepheid, USA) and "line probe assays" LiPA - Genotype ${ }^{\circledR}$ MTBDRsl (MTBDRsl) (Hain Lifescience, Nehren, Germany) for rapid $\mathrm{TB}$ and MDR-TB diagnosis worldwide (WHO, 2017).

Xpert ${ }^{\circledR}$ MTB/RIF assay is based on polymerase chain reaction (PCR) amplification, followed by detection of mutations in its Rifampicin ResistanceDetermining Region (RRDR). Furthermore, most isolates resistant to Rifampicin (RIF) are also resistant to Isoniazid (INH), therefore the presence of rpoB mutations can be used as a surrogate marker for MDRTB (WHO, 2016). Genotype ${ }^{\circledR}$ MTBDRsl (MTBDRsl) is a rapid DNA-based test for detecting specific mutations associated with resistance to fluoroquinolones and
Second-Line Injectable Drugs (SLIDs) in Mycobacterium tuberculosis complex (WHO, 2017).

Tuberculosis has become a health problem reaching the stage of epidemic proportion in South Africa (Evans, 2011). Factors such as inadequate chemotherapy, poor drug quality, poor adherence to treatment, treatment failure, prior treatment, cavity pulmonary TB, HIV infection and diabetes accounted for the development of drug resistance in TB (Akl and Mahalli, 2012; Caminero, 2010).

South Africa has made notable progress in improving TB control, but the burden of TB remains enormous. Treatment success rate for adult MDR-TB and XDR-TB remained low at 42 and $18 \%$ respectively for those diagnosed in 2010 (Churchyard et al., 2014).

The Eastern Cape (EC) Province is considered as a home to some of the poorest and most vulnerable communities in South Africa. Access to basic medical services in these areas of the region is limited, making provision of health care a big problem for these communities and also making information about treatment outcomes and risk factors associated to drug resistant tuberculosis limited. Therefore, the aim of this study was to determine treatment outcomes and risk factors associated with drug resistant tuberculosis.

\section{Materials and Methods}

\section{Study Design}

This study was an observational descriptive study. Samples were prospectively collected from June 2015 to September 2017.

\section{Study Settings and Sample Collection}

The study was conducted from 2 sub-districts in Eastern Cape: King Sabata Dalindyebo (KSD) and Mhlontlo. Gateway clinic in KSD has a satellite MDRTB clinic able to manage all cases suspected of being drug-resistant tuberculosis (DR-TB) cases from KSD and Mhlontlo sub-districts. Medical records from all DR-TB cases are also kept at Gateway clinic while all clinical samples are sent to Mthatha at the National Health Laboratory Services (NHLS) for analysis. Samples were collected by patients (Sputum) and by medical doctors (CSF, Pleural fluid) as part of the patients' routine management and submitted to the NHLS TB laboratory.

On its own, Gateway clinic manages MDR-TB suspects referred from 57 Primary Health Care (PHC) facilities: 33 facilities from KSD and 24 facilities from Mhlontlo. Gateway clinic performs the following functions: initiation of MDR-TB treatment, monthly monitoring and follow up of patients (conducting DRTB reviews on Wednesdays and Thursdays weekly) whilst radiology service is outsourced from Mthatha General Hospital. The clinic also has Mobile injection and Directly Observed Treatment (DOTS) teams. 


\section{Laboratory Diagnosis of MDR-TB and XDR-TB}

\section{Molecular Methods}

Xpert ${ }^{\circledR}$ MTB/RIF assay (Cepheid, Sunnyvale, USA): The GeneXpert was performed directly on TB samples using the newer version (G4) of cartridges according to the manufacturer's recommendations. Samples were decontaminated and reagent buffer containing $\mathrm{NaOH}$ and isopropanol was added at the ratio of 2:1, followed by incubation at room temperature for $15 \mathrm{~min}$. Two millilitres of the final samples were then transferred into the Xpert MTB/RIF cartridge and after mixing, the cartridge was loaded into the GeneXpert instrument. Reported results were M. tuberculosis negative or positive and Rifampicin (RIF) resistant or susceptible (Xpert ${ }^{\circledR}$ MTB/RIF detects mutations in Rifampicin Resistance-Determining Region of M.tb).

LiPA (Hain Lifescience, Nehren, Germany): Line probe assays are designed to identify $M$. tuberculosis complex and simultaneously detect mutations associated with drug resistance. We used in this study MTBDRsl version 2.0. This is designed for simultaneous detection of the most important rpoB mutations, which confer RIF resistance and katG and inhA mutations, which confer high-level and low level isoniazid (INH) resistance, respectively. In addition, MTBDRsl detects specific mutations associated with resistance to the fluoroquinolones (FQs) (including ofloxacin, moxifloxacin, levofloxacin and gatifloxacin) and secondline injectable drugs (SLIDs) (including kanamycin, amikacin and capreomycin) in M. tuberculosis complex species. Version 2.0 detects mutations in the gyrA quinolone resistance-determining region (codons 88, 90, 91, 94) and rrs (codons 1401, 1402, 1484), mutations in the gyrB quinolone resistance-determining region (codons 538, 540) and the eis promoter region (codons $37,-14,-12,-10,-2)$ (WHO, 2017; 2016).

\section{Phenotypic Drug Susceptibility Testing (DST)}

Phenotypic Drug Susceptibility Testing (DST) was carried out using the automated BACTEC Mycobacterial Growth Indicator Tube (MGIT) 960 (Becton Dickinson, USA). These tests are currently regarded as the "gold standard" for drug susceptibility testing and were used to control the follow up of patients' progress and their different treatment outcomes. MGIT960 is an in-vitro diagnostic instrument designed and optimized for the rapid detection of mycobacteria from clinical specimens (except blood). The results indicating susceptibility or resistance were interpreted and reported automatically by the MGIT system using predefined algorithms that compare bacterial growth in the drug-containing tube with the growth in the drug-free control tube.
The following critical concentrations for 1st and 2nd line anti-TB drugs were used when testing using MGIT960: Isoniazid $(0.1 \mu \mathrm{g} / \mathrm{mL}$ of medium), Rifampin $(1.0 \mu \mathrm{g} / \mathrm{mL}$ of medium), Ofloxacin $(2.0 \mu \mathrm{g} / \mathrm{mL})$, Amikacin $(1.0 \mu \mathrm{g} / \mathrm{mL})$, Kanamycin $(2.5 \mu \mathrm{g} / \mathrm{mL})$ and Capreomycin $(2.5 \mu \mathrm{g} / \mathrm{mL})$.

\section{Treatment and Management of DR-TB Patients}

Any DR-TB patient received was directly transferred to MDR-TB satellite clinic at Gateway clinic for appropriate management. Each patient was treated with a personalised regimen composed of second line injectable drugs (kanamycin, amikacin, or capreomycin), fluoroquinolones (levofloxacin, ofloxacin or moxifloxacin), ethambutol, pyrazinamide, high dose isoniazid. The treatment's duration ranged from 6 to 18 months for patients with MDR-TB and 24 months for patients with XDR-TB. For MDR-TB treatment, the short regimen was the following: 4-6 months of Kanamycin (Km) -Moxifloxacin (Mfx) -Prothionamide (Pto) -Clofazimine (Cfz) -Pyrazinamide (Z) -high-dose Isoniazid (H)-Ethambutol (E)/5 months of Mfx-Cfz-Z-E; and long regimen: 6 months of: Z-Km(Am)-Mfx-EtoTerizidone (Trd)/18 months of: Z-Mfx-Eto-Trd.

For XDR-TB treatment: 6 months of Cm-Mfx-EtoTrd -Z-PAS-Clofazimine and 18 months of Mfx-Eto-Trd or Cs-Z-PAS/Clofazimine.

\section{Treatment Outcomes}

Drug resistant tuberculosis patient files were kept at Mthatha Gateway clinic and were accessed by the researchers; it's only when patients were transferred out (for example XDR-TB patients transferred in PortElizabeth and East-London for hospitalization) that they were allowed to leave with their files. For the purpose of this study, treatment outcomes were recorded as "cure", "treatment complete", "default", "relapse", "death", or "transfer out". According to WHO recommendations (WHO, 2014), treatment outcomes were defined as follows:

- Cure: A patient who has converted and has remained TB culture negative, has completed treatment and has been consistently culture-negative for five consecutive months in the final twelve months of treatment who also had 2 or more consecutive negative cultures, taken at least thirty days apart.

- Treatment complete: A patient who has completed treatment but does not meet the definition for "cure" due to lack of bacteriologic results (i.e., less than five cultures were performed in the final twelve months of treatment).

- Death: A patient who dies from any cause while on DR-TB treatment.

- Treatment default: A patient who interrupts DR-TB treatment for two or more consecutive months for any reason. 
- Relapse: A patient who was declared cured after treatment but who reported back to the health service and is now found to be sputum smear-positive.

- Transfer out: A patient who has been transferred to a reporting unit in another province or within the same province and for whom the treatment outcome is unknown.

Finally, the above treatment outcomes were classified in 2 categories: (i) Favourable outcomes included "cure", "treatment complete" and "patients who are still on treatment while strictly adhering to their anti-TB therapy"; (ii) poor outcomes included "default", "relapse", "death" and "transfer out".

\section{DR-TB Risk Factors}

Risk for developing DR-TB was assessed. Specific elements of the history that suggested an increased risk for drug resistance consisted of: HIV status, CD4 count, Viral load (VL), gender, Age and patient's previous exposure to anti-TB therapy.

\section{Data Variables and Analysis}

Statistical Package for the Social Sciences (SPSS) version 22.0 was used for all statistical analyses, the level of significance was fixed at $\rho \square 0.05$. Continuous variables were expressed as mean \pm Standard Error of the Mean (SEM) and categorical variables were expressed as proportions $(\%)$.

\section{Results}

From July 2015 to September 2017, a total number of 224 patients were enrolled in the study. The mean age of participants was $38.2( \pm 15.2)$, ranging from 3 to 85 years. Men accounted for $122(54.5 \%)$ with a mean age of $38.6( \pm 12.9)$ ranging from 3 to 72 years and females for $102(45.5 \%)$ with a mean age of $37.8( \pm 17.6)$ ranging from 6 to 85 years.

Table 1: General characteristics of participants enrolled in the study $(n=224)$

\begin{tabular}{ll}
\hline Variable of interest & Number $\mathrm{n}(\%)$ \\
\hline Gender & $122(54.5)$ \\
Male & $102(45.5)$ \\
Female & \\
Age $($ Years $)($ mean \pm SEM) & $104(46.4)$ \\
$<35$ & \\
$(25.8 \pm 0.6)$ & $104(46.4)$ \\
$35-65$ & $16(7.1)$ \\
$(45.5 \pm 0.8)$ & \\
$>65$ & \\
$(71.6 \pm 1.5)$ & $76(33.9)$ \\
HIV status & $148(66.1)$ \\
HIV (-) & \\
HIV (+) & $194(86.6)$ \\
Sub-district of origin & $30(13.4)$ \\
KSD & \\
Mhlontlo &
\end{tabular}

Of 224 patients, $76(33.9 \%)$ were HIV negative and $148(66.1 \%)$ were HIV positive as described in Table 1. Of 122 male participants, 77 (63.1\%) were HIV positive and $45(36.9 \%)$ were HIV negative. However, from 102 female participants, $71(69.6 \%)$ were HIV positive and 31 (30.4\%) were HIV negative.

The above described patients were referred from 2 sub-districts: KSD and Mhlontlo, of which $86.6 \%$ were coming from KSD and 13.4\% from Mhlontlo.

\section{Risk Factors and Outcomes of MDR-TB and XDR- TB Patients}

Of 224 TB isolates obtained, 196 (87.5\%) were MDR-TB (including 23 rifampicin mono-resistant isolates), $2(0.9 \%)$ were isoniazid mono-resistant and $26(11.6 \%)$ were Pre-XDR and XDR-TB. Although $57.7 \%$ of females developed XDR-TB as compared to $42.3 \%$ males, this difference did not reach significant difference $(\mathrm{OR}=1.7 ; \mathrm{CI}: 0.8-3.9 ; \mathrm{p}=0.13)$ as shown in Table 2.

Data on patients' outcomes were analysed for 222 patients (2 INH mono-resistant patients were excluded), of which 196 MDR-TB and 26 XDR-TB.Of 196 MDRTB patients, $143(72.9 \%)$ had a favourable outcome post anti-TB therapy as compared to $17(65.4 \%)$ XDR-TB patients out of 26; but this result did not achieve significant difference $(\mathrm{OR}=1.5$; CI: $0.6-3.4 ; \rho=0.27)$.

The study has shown that whilst $118(68.6 \%)$ out of 172 MDR-TB cases were newly diagnosed, previous exposure to anti-TB therapy was found to be a significant risk factor for developing XDR-TB (OR = 2.5; CI $=1.1-5.9 ; \rho=0.023)$ as displayed in Table 2 .

HIV status, CD4 count, HIV Viral Load (VL) and demographic factors (age and gender) were not significantly associated with the development of DR-TB (Table 2).

As described in Table 3, despite that 34 (26.2\%) out of 130 new drug-resistant (DR)-TB patients completed their therapy, only $17(13.1 \%)$ were declared cured as compared to $23(33.8 \%)$ cured patients among the previously treated group $(\rho<0.0001)$. In contrary, 9 $(13.2 \%)$ cases of relapse and $11(16.2 \%)$ defaulters were found among the previously treated DR-TB patients as compared to new cases [1 $(0.8 \%)$ relapse versus 11 $(8.5 \%)$ defaulters, respectively] on therapy $(\rho<0.0001)$. In addition, 8 out of 130 new patients $(6.2 \%)$ were transferred out as compared to only 1 out of 68 of previously treated patients $(1.5 \%)(\rho<0.0001)$. The difference in mortality rates between patients with previous history of anti-TB therapy $(7.4 \%)$ and treatment-naive counterparts $(6.2 \%)$ seemed not to reach statistical significance. 
Table 2: Outcomes and risk factors associated with DR-TB

\begin{tabular}{|c|c|c|c|}
\hline & MDR-TB $(\mathrm{n}=196)^{*}$ & XDR-TB $(\mathrm{n}=26)^{*}$ & P-value \\
\hline \multicolumn{4}{|l|}{ Gender*: } \\
\hline Male & $110(56.1 \%)$ & $11(42.3 \%)$ & \multirow[t]{2}{*}{0.13} \\
\hline Female & $86(43.9 \%)$ & $15(57.7 \%)$ & \\
\hline \multicolumn{4}{|l|}{ Age*: } \\
\hline$>35$ & $92(46.9 \%)$ & $10(38.5 \%)$ & \multirow[t]{3}{*}{0.55} \\
\hline $35-65$ & $91(46.4 \%)$ & $13(50 \%)$ & \\
\hline$>65$ & $13(6.6 \%)$ & $3(11.5 \%)$ & \\
\hline \multicolumn{4}{|l|}{ Patient's history**: } \\
\hline New & $118 / 172(68.6 \%)$ & $12 / 26(46.2 \%)$ & \multirow[t]{2}{*}{0.023} \\
\hline Previously treated & $54 / 172(31.4 \%)$ & $14 / 26(53.8 \%)$ & \\
\hline \multicolumn{4}{|l|}{ HIV status $* *$ : } \\
\hline HIV negative & $62 / 193(32.1 \%)$ & $8 / 25(32 \%)$ & \multirow[t]{2}{*}{0.59} \\
\hline HIV positive & $131 / 193(67.9 \%)$ & $17 / 25(68 \%)$ & \\
\hline \multicolumn{4}{|l|}{ Outcomes*: } \\
\hline Favorable outcomes & $143(72.9 \%)$ & $17(65.4 \%)$ & \multirow[t]{2}{*}{0.27} \\
\hline Poor outcomes & $53(27 \%)$ & $9(34.6 \%)$ & \\
\hline \multicolumn{4}{|l|}{ CD4 count**: } \\
\hline$<200$ & $50 / 101(49.5 \%)$ & $8 / 17(47.1 \%)$ & \multirow[t]{3}{*}{0.36} \\
\hline $200-349$ & $24 / 101(23.8 \%)$ & $2 / 17(11.8 \%$ & \\
\hline$\geq 350$ & $27 / 101(26.7 \%)$ & $7 / 17(41.2 \%)$ & \\
\hline \multicolumn{4}{|l|}{ Viral load**: } \\
\hline$<20$ & $29 / 81(35.8 \%$ & $3 / 15(20 \%)$ & \multirow[t]{2}{*}{0.18} \\
\hline$\geq 20$ & $52 / 81(64.2 \%)$ & $12 / 15(80 \%)$ & \\
\hline
\end{tabular}

(*For gender, age and outcomes: we had 196 MDR-TB and 26 XDR-TB patients.**For the rest of variables, available data were as follows: patients' history - 172 MDR-TB and 26 XDR-TB; HIV status - 193 MDR-TB and 25 XDR-TB; CD4 count - 101 MDRTB and 17 XDR-TB; viral load - 81 MDR-TB and 15 XDR-TB patients)

Table 3: Treatment outcomes of MDR-TB and XDR-TB patients stratified by new and previous anti-TB treatment in Mthatha, South Africa

\begin{tabular}{llll}
\hline & Patient's category & & \\
Treatment outcomes & New anti-TB treatment $(\mathrm{n}=130)$ & Previously treated $(\mathrm{n}=68)$ & \\
\hline $\begin{array}{l}\text { Outcomes } \\
\text { Favourable outcomes }\end{array}$ & & & \\
Cured & $17(13.1 \%)$ & $23(33.8 \%)$ \\
Treatment complete & $34(26.2 \%)$ & $10(14.7 \%)$ \\
Still on anti-TB therapy & $50(38.5 \%)$ & $9(13.2 \%)$ \\
Poor outcomes & $11(8.5 \%)$ & $11(16.2 \%)$ \\
Defaulter & $1(0.8 \%)$ & $9(13.2 \%)$ \\
Relapse & $8(6.2 \%)$ & $1(1.5 \%)$ \\
Transfer out & $8(6.2 \%)$ & $5(7.4 \%)$ \\
Died & &
\end{tabular}

\section{Discussion}

In this present study, many XDR-TB patients had poor outcomes as compared to MDR-TB patients and that previously treated patients had increased risk of developing XDR-TB. Similar findings were also reported by Masjedi et al. (2010) in their study in Iran, reported that poor treatment outcome was mostly related to patients with XDR-TB (Masjedi et al., 2010); in Estonia, Kliiman and Altraja (2009) described that patients with XDR-TB had poor outcomes compared to patients with MDR-TB and they also reported that previously treated patients had increased risk of developing XDR-TB (Kliiman and Altraja, 2009). Smith et al. (2015), in Russia, found that poor outcomes were associated with the emergence of extensive drug resistant TB.
In our study, among DR-TB (MDR-TB and XDRTB) patients, we recorded a combined low cure rate of $39.3 \%$ despite that $40.9 \%$ of patients completed their anti-TB treatment. This result is in contrary to a study by Reechaipichitkul et al. (2014) who reported a cure rate of $72.4 \%$ while having only $1.8 \%$ of patients who were not declared cured after completing treatment.

We also found in this present study that defaulters and relapsing patients were significantly associated with the history of previous anti-TB treatment. Our results are once more not keeping with findings published by Reechaipichitkul et al. (2014) who identified that 87.5\% of their DR-TB patients who defaulted and $68.42 \%$ patients who relapsed did not have a significant history of previous anti-TB therapy. 
Among the previously treated patients, we had high rates of patients who defaulted and those who relapsed. However, we had a favourable cure rate of $33.8 \%$ of previously treated patients as compared to $13.1 \%$ newly diagnosed DR-TB patients. This is not in line with a study conducted in Latvia that reported XDR-TB cure rate of $50 \%$ by Kuksa et al. (2014). The authors also reported that XDR-TB patients in their study not only had a higher risk of treatment failure compared with MDR-TB patients (29\% Vs. $8 \%$, respectively, $\mathrm{P}<0.001)$ but they also found that unfavourable treatment outcomes were significantly associated with patients who were previously treated (Kuksa et al., 2014).

In the past decade, Drug-resistant tuberculosis has traditionally been thought to develop as a result of selection pressure that occurs with inadequate treatment of tuberculosis, incomplete adherence to treatment, or sub therapeutic drug levels (Shah et al., 2017). However, patients tend to acquire directly resistance from other MDR-TB patients without the need of having any previous history of treatment with TB. In our study, $68.6 \%$ of patients were MDR-TB newly diagnosed and $46.2 \%$ were new XDR-TB patients. Dheda et al. (2010) in Cape Town reported that the majority of new DR-TB cases diagnosed in SA were due to transmission of already resistant strains. The Eastern Cape Province has the 3rd largest population with close to 7 million people and is the 2nd poorest province in South Africa and has the 2 nd lowest percentage of people $(10.2 \%)$ with access to a medical aid in the country. In 2010, The Eastern Cape was the second worst affected province with drug resistant TB after KwaZulu-Natal with more than $10 \%$ of strains having resistance to at least 1 drug NDOH, 2013. In Mthatha and surroundings where this study was conducted, we typically have congregated wards and crowded clinics. In addition to healthcare facilities where patients spend time together, it is possible that direct TB transmission may also occur in schools, churches and taxis. Similar findings were reported by Shah et al. (2017) in KwaZulu-Natal, who identified epidemiologic links among $30 \%$ of their participants and these networks included multiple households and hospitals, in addition to person-to-person links among schoolmates and church members. Although transmission of drugresistant tuberculosis in hospitals is well described (Wells et al., 2007), interventions that decrease transmission in other community settings are urgently needed. Finally, it is also important to underline that approximately $1.8 \%$ of new TB cases and $6.7 \%$ of previously treated TB cases in SA are Multidrug Resistant (MDR) (WHO, 2014).

The present study confirms that previous anti-TB treatment significantly increases the risk of having XDR-TB. Dalton et al. (2012) showed that previous treatment was consistently the strongest risk factor for resistance to these drugs, increasing the risk of XDRTB more than fourfold.
Patients with a history of previous treatment is a huge problem nowadays, they often have the most complex cases to manage. They constitute a very heterogeneous group that is composed of patients with TB who experience relapse after receiving successful treatment, those who return after default and those who start receiving a re-treatment regimen after having experienced previous treatment failure, as well as other patients such as those who received unknown or unstandardized treatment regimens (Zignol et al., 2007). Because of their high risk of harbouring drug-resistant strains, previously treated patients require more complex laboratory investigations to diagnose their disease and much longer, more expensive and more potentially toxic regimens to be cured (Zignol et al., 2007). More effort should be put on the management of those patients, hence previously treated TB should no longer be ignored and should receive the same type of high-quality care that is promoted for patients with newly diagnosed cases.

A total of $57.7 \%$ of female participants in this study were XDR-TB patients (versus $42.3 \%$ males). In Ukraine, Aibana et al. (2017) reported similar findings. In 2009, according to a study done in Georgia by Lomtadze et al. (2009), women were independent risk factors for the presence of MDR-TB. Another study in South Africa found higher percentages of XDR-TB (50\%-56\%) than MDR-TB patients (43\%-53\%) among women (Heller et al., 2009). Taking into consideration the above mentioned information, we hypothesized that this association could be related to the fact that women care for men and are more exposed to DR-TB both in households and in healthcare settings. Women are also more likely to participate in formal and informal care works, with potential exposure to drug-resistant TB strains and therefore primary DR-TB might be more likely to develop (O'Donnell et al., 2011). It can also be due to the fact that some of the anti-DR-TB drugs are used for the treatment of sexually transmitted diseases leading to resistance - for example resistance to amikacin, a commonly used aminoglycoside in South Africa for the treatment of other infectious diseases (i.e., infections caused by Gram negative bacteria) in addition to the treatment of MDR-TB, induces almost complete cross-resistance to kanamycin (Sugawara et al., 2009).

HIV-related factors could explain the association of gender differences in drug-resistant $\mathrm{TB}$, for example, women with drug-resistant TB may be more adherent to Antiretroviral Therapy (ART), leading to improved survival and therefore increased time for DR-TB to develop (O'Donnell et al., 2011); another factor associated with secondary development of DR-TB, such as TB medication adherence or previous MDR-TB treatment, could explain the association between XDRTB and female gender. 


\section{Limitations}

Limitations for this study include: (i) small number of pre-XDR and XDR-TB cases as compared to exclusively MDR-TB patients; (ii) absence of the cohort of susceptible TB for comparison with DR-TB since we worked in DR-TB clinic only; and (iii) missing of some clinical and/or laboratory findings that led to the exclusion of some cases during analysis.

\section{Conclusion and Recommendations}

With the increasing number of DR-TB cases around the world, the epidemic caused by Mycobacterium tuberculosis is recognized as a threat to global health. The present study has raised important implications toward DR-TB control efforts in Mthatha, Eastern cape province of South Africa:

- MDR-TB patients had more favourable outcomes than XDR-TB patients following appropriate antiTB therapy.

- High rates of DR-TB were diagnosed among treatment-naive patients pointing to the importance of DR-TB acquisition through direct transmission rather than the evolution of resistance.

- Previously treated patients had increased risk of developing XDR-TB.

- Prevalent cases of relapses and defaulters were seen among previously treated patients.

- Females were more likely to contract and develop XDR-TB than males.

The following recommendations for the EC department of health and healthcare facilities can be considered:

1. Intensify DR-TB case findings.

2. Strengthen DR-TB infection prevention and control program in healthcare facilities.

3. Ensure uninterrupted supply of high quality anti-TB drugs.

4. Regular monitoring and evaluation of the DR-TB program.

5. Promote the use of individualized DR-TB regimen according to the susceptibility test result of each patient.

6. Continuous assessment by means of surveillance of DR-TB among new patients.

7. Strengthen DR-TB patients' education and information regarding their conditions and management.

8. Implement infection prevention and control measures among households and in the communities.

9. Strengthen screening program among households where a relative is diagnosed with DR-TB.

10. Strengthen adherence to anti-TB therapy.

\section{Acknowledgments}

The authors would like to thank Dr. Lwazi Dumke and the staff at Gateway clinic in Mthatha for their cooperation and help during this study.

\section{Author's Contributions}

Carine Bokop Fotso: Participated in all experiments, coordinated the data-analysis and contributed to the writing of the manuscript.

Sandeep D. Vasaikar: Contributed in study design and final proof reading of the manuscript.

Teke Apalata: Designed the research plan, organized the study, contributed in data analysis and edited the final manuscript.

\section{Ethics}

Ethical approval was obtained from the Research Ethics and Biosafety Committee of Walter Sisulu University (Ref. No: 088/2016) whilst permissions to conduct the study were obtained from the Eastern Cape Department of Health (No: EC_201710_005) and from the National Health Laboratory Services (NHLS). In order to protect privacy and confidentiality of the patients, no names were recorded and instead, a personalized research number was used for each patient and only the main investigator had access to the collected data.

\section{References}

Aibana, O., M. Bachmaha, V. Krasiuk, N. Rybak and T.P. Flanigan et al. 2017. Risk factors for poor multidrug-resistant tuberculosis treatment outcomes in Kyiv Oblast, Ukraine. BMC Infec. Dis., 17: 129. DOI: 10.1186/s12879-017-2230-2

Akl, M.A. and A.A. Mahalli, 2012. Drug resistant tuberculosis: Risk factors and resources-utilization at a chest disease clinic, Alexandria. Egypt J. Am. Sci., 8: 107-12.

Bateman, C., 2015. Eastern Cape treatment dysfunction boosts virulent new XDR-TB strain. South African Medical J., 105: 165-7. DOI: 10.7196/SAMJ.9475

Caminero, J.A., 2010. Multidrug-resistant tuberculosis: Epidemiology, risk factors and case finding. Int. J. Tuberculosis Lung Disease, 14: 382-90.

CDC. CDC 24/7: Saving Lives Protecting People, 2016. Division of tuberculosis elimination. Centers for disease control and prevention.

Churchyard, G.J., L.D. Mametja, L. Mvusi, N. Ndjeka and A.C. Hesseling et al., 2014. Tuberculosis control in South Africa: Successes, challenges and recommendations. South African Medical J., 104: 244-8. 
Dalton, T., P. Cegielski, S. Akksilp, L. Asencios and J. Campos Caoili et al., 2012. Prevalence of and risk factors for resistance to second-line drugs in people with multidrug-resistant tuberculosis in eight countries: A prospective cohort study. Lancet, 380 : 1406-17. DOI: 10.1016/S0140-6736(12)60734-X

Dheda, K., K. Shean, A. Zumla, M. Badri and E.M. Streicher et al., 2010. Early treatment outcomes and HIV status of patients with extensively drugresistant tuberculosis in South Africa: A retrospective cohort study. Lancet. 375: 1798-807. DOI: $10.1016 / \mathrm{S} 0140-6736(10) 60492-8$

Evans, C.A., 2011. GeneXpert--a game-changer for tuberculosis control? PLoS Medicine, 8: e1001064.

Heller, T., R.J. Lessells, C.G. Wallrauch, T. Barnighausen and G.S. Cooke et al., 2010. Community-based treatment for multidrug-resistant tuberculosis in rural KwaZulu-Natal, South Africa. Int. J. Tuberculosis Lung Disease, 14: 420-6.

Kliiman, K. and A. Altraja, 2009. Predictors of poor treatment outcome in multi- and extensively drugresistant pulmonary TB. Eur. Respiratory J., 33: 1085-94. DOI: 10.1183/09031936.00155708

Kuksa, L, V. Riekstina, V. Leimane, I. Ozere and G. Skenders et al., 2014. Multi- and extensively drugresistant tuberculosis in Latvia: Trends, characteristics and treatment outcomes. Public Health Action, 4: S47-53.

DOI: $10.5588 /$ pha. 14.0041

Lomtadze, N., R. Aspindzelashvili, M. Janjgava, V. Mirtskhulava and A. Wright et al., 2009. Prevalence and risk factors for multidrug-resistant tuberculosis in the Republic of Georgia: A population-based study. Int. J. Tuberculosis Lung Disease, 13: 68-73.

Masjedi, M.R., P. Tabarsi, P. Baghaei, S. Jalali and P. Farnia et al., 2010. Extensively drug-resistant tuberculosis treatment outcome in Iran: A case series of seven patients. Int. J. Infectious Diseases, 14: e399-402. DOI: 10.1016/j.ijid.2009.07.002

O'Donnell, M.R., J. Zelnick, L. Werner, I. Master and M. Loveday et al., 2011. Extensively drug-resistant tuberculosis in women, KwaZulu-Natal, South Africa. Emerging Infectious Diseases, 17: 1942-5. DOI: $10.3201 /$ eid1710.110105

Reechaipichitkul, W., A. So-Ngern and P. Chaimanee, 2014. Treatment outcomes of new and previouslytreated smear positive pulmonary tuberculosis at Srinagarind Hospital, a tertiary care center in northeast Thailand. J. Medical Association Thailand, 97: 490-9.

Ryan, K.J. and C.G. Ray, 2004. Mycobacteria. Sherris Medical Microbiology: An Introduction to Infectious Diseases New York: McGraw-Hill, pp: 439.
Schaaf, H.S., P. Botha, N. Beyers, R.P. Gie and H.A. Vermeulen et al., 1996. The 5-year outcome of multidrug resistant tuberculosis patients in the Cape Province of South Africa. Tropical Medicine International Health, 1: 718-22. DOI: $10.1111 /$ j.1365-3156.1996.tb00100.x

Shah, N.S., S.C. Auld, J.C. Brust, B. Mathema and N. Ismail et al., 2017. Transmission of extensively drug-resistant tuberculosis in South Africa. New England J. Med., 376: 243-53.

DOI: 10.1056/NEJMoa1604544

Smith, S.E., J. Ershova, N. Vlasova, E. Nikishova and I. Tarasova et al., 2015. Risk factors for acquisition of drug resistance during multidrug-resistant tuberculosis treatment, Arkhangelsk Oblast, Russia, 2005-2010. Emerging Infec. Diseases, 21: 1002-11. DOI: $10.3201 /$ eid2106.141907

STATSSA, 2013. Mortality and causes of death in South Africa: Findings from death notification. Statistics South Africa.

Sugawara, I., J. Zhang and C. Li, 2009. Cross-resistance of Mycobacterium tuberculosis isolates among streptomycin, Kanamycin and Amikacin. Indian J. Experimental Biology, 47: 520-2.

Wells, C.D., J.P. Cegielski, L.J. Nelson, K.F. Laserson and T.H. Holtz et al., 2007. HIV infection and multidrug-resistant tuberculosis: The perfect storm. J. Infect. Diseases, 196: S86-107. DOI: $10.1086 / 518665$

WHO, 2006. Factsheet tuberculosis. World Health Organisation.

WHO, 2010. Multidrug and extensively drug-resistant TB (M/XDR-TB): 2010 global report on surveillance and Response WHO/HTM/TB/2010, World Health Organization.

WHO, 2012. Global tuberculosis report 2012. WHO/HTM/TB/2012.6. Geneva: WHO.

WHO, 2014. Global Tuberculosis Report 2014. Geneva: WHO.

WHO, 2015. Use of high burden country lists for tuberculosis by WHO in the post-2015 era. Programme WHOgt, Geneva, Switzerland.

WHO, 2016. WHO treatment guidelines for drugresistant tuberculosis. WHO Treatment Guidelines for Drug-Resistant Tuberculosis, World Health Organization.

WHO, 2017. The impact of the roll-out of rapid molecular diagnostic testing for tuberculosis on empirical treatment in Cape Town, South Africa. Bulletin WHO, 95: 545-608.

Zignol, M., A. Wright, E. Jaramillo, P. Nunn and M.C. Raviglione, 2007. Patients with previously treated tuberculosis no longer neglected. Clinical Infec. Diseases, 44: 61-4. DOI: 10.1086/509328 